\title{
A New Group-Training Procedure for Habituation Demonstrates That Presynaptic Glutamate Release Contributes to Long-Term Memory in Caenorhabditis elegans
}

\author{
Jacqueline K. Rose, Karla R. Kaun, and Catharine H. Rankin ${ }^{1}$ \\ Department of Psychology and Brain Research Centre, University of British Columbia, Vancouver, British Columbia V6T 1Z4, Canada
}

\begin{abstract}
In the experiments reported here we have developed a new group-training protocol for assessing long-term memory for habituation in Caenorbabditis elegans. We have replicated all of the major findings of the original single-worm protocol using the new protocol: (1) distributed training produced long-term retention of training, massed training did not; (2) distributed training at long interstimulus intervals (ISIs) produced long-term retention, short ISIs did not; and (3) long-term memory for distributed training is protein synthesis-dependent as it could be blocked by heat shock during the inter-block interval. In addition, we have shown that long-term memory for habituation is graded, depending on the number of blocks of stimuli in training. The inter-block interval must be $>40 \mathrm{~min}$ for long-term retention of training to occur. Finally, we have tested long-term memory for habituation training in a strain of worms with a mutation in a vesicular glutamate transporter in the sensory neurons that transduce tap (eat-4). The results from these eat-4 worms indicate that glutamate release from the sensory neurons has an important role in the formation of long-term memory for habituation.
\end{abstract}

Research using a number of different organisms has shown that if a stimulus is repeatedly presented over time, subjects will make fewer and/or smaller responses to the stimulus. This is termed habituation (Groves and Thompson 1970). Under some conditions, this decreased level of responding can still be seen hours (Beck and Rankin 1997), days (Tully et al. 1994), and weeks (Carew et al. 1972) after the original repeated exposure to the stimulus. This retention of habituation training is termed long-term habituation.

One model system that has been studied recently in an attempt to investigate the characteristics and mechanisms of this long-term memory for habituation is the nematode Caenorhabditis elegans. Beck and Rankin (1997) showed that distributed, or spaced, habituation training of the response to tap in C. elegans resulted in retention of the habituated response $24 \mathrm{~h}$ later, whereas massed training did not. In addition, only worms that received distributed training at long interstimulus intervals (ISI; $60 \mathrm{sec}$ ) demonstrated long-term retention of the habituation training; worms that received distributed habituation training at a short ISI (10 sec) showed no retention 24 h later.

Because remarkably little is known about the biological foundations of long-term habituation, one of the objectives of the current study is to develop $C$. elegans as a model

${ }^{1}$ Corresponding author.

E-MAIL crankin@psych.ubc.ca; FAX (604) 822-6923.

Article and publication are at http://www.learnmem.org/cgi/doi/ $10.1101 / \mathrm{lm} .46802$. system in which to investigate the cellular mechanisms of long-term memory for habituation training. In our previous studies of long-term memory, C. elegans were individually trained with three blocks of training (20 trains of taps at a 60 -sec ISI) separated by 1 -h rest periods (taking $\sim 4 \mathrm{~h}$ per worm) and then tested $24 \mathrm{~h}$ later with 20 trains of taps at a 60-sec ISI (Beck and Rankin 1995, 1997). Although this procedure was successful in producing long-term habituation, it was very time-consuming. The present series of experiments tested whether a new protocol, in which groups of worms were trained simultaneously with four blocks of training (20 single taps at a 60-sec ISI) followed by 1-h rest periods, would lead to long-term memory when worms were tested with 10 single taps at a 60 -sec ISI $24 \mathrm{~h}$ later. This study also tests the efficacy of the new protocol in screening for mutations that affect long-term memory in $C$. elegans by testing for long-term memory using both the old and new protocols with a mutant strain, eat-4, which shows altered short-term habituation (Rankin and Wicks 2000).

\section{RESULTS}

\section{The New Habituation Protocol Replicates the Findings of the Original Protocol}

The first stage of establishing the new group-training protocol for studying long-term memory for habituation training in C. elegans was to replicate the findings of Beck and Rankin (1995, 1997). The protocol used in Beck and Rankin

LEARNING \& MEMORY 9:130-137 @ 2002 by Cold Spring Harbor Laboratory Press ISSN1072-0502/02 \$5.00

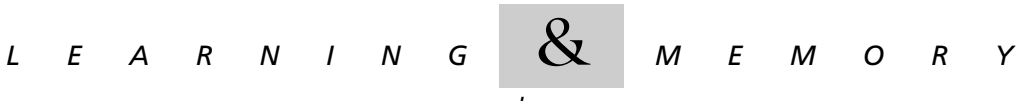

$$
\begin{aligned}
& \text { www.learnmem.org }
\end{aligned}
$$


(1995, 1997) consisted of three blocks of distributed training using "trains of taps" as the habituation stimulus, whereas the new protocol consists of an additional fourth block of training and employed single taps as the habituation stimulus. In the first experiment with the new protocol, the effects of massed and distributed training were tested. There were four groups of wild-type worms tested: a distributed training group (four blocks of 20 single taps at a 60-sec ISI separated by $1 \mathrm{~h} ; n=27)$ and its matched control group $(n=24)$ as well as a massed training group (one block of 80 single taps at a 60 -sec ISI; $n=22$ ) with its tap control group ( $n=22)$. The results can be seen in Figure 1 in which both the mean responses to each of the 10 test taps and the overall mean response data for the test stimuli are shown. In the graph showing the responses to the 10 test taps for the distributed training, responses from trained worms were all lower than the responses for the untrained control group (Fig. 1A), whereas there was great overlap between responses of trained and untrained worms in the massed condition (Fig. 1B). The overall mean response to the 10 test single taps by the distributed group was significantly smaller than the mean response to the 10 test single taps for the matched tap control group $(t(49)=3.03, P<0.01$; Fig. 1C). When the mean response to the test taps was compared for the massed trained group and its matched tap control group, there was no significant difference in response magnitudes $(t(42)=0.52, P>0.10)$. These results replicate the findings of Beck and Rankin (1997); distributed training produced long-term memory for habituation, whereas massed training did not.

Beck and Rankin (1997) also found that worms that received distributed long-term habituation training at a short (10-sec) ISI did not show retention of the habituation training $24 \mathrm{~h}$ later. The next experiment tested whether delivering stimuli at a 10 -sec ISI in the new group-training protocol would also fail to produce long-term memory. The procedure for this experiment was the same as the group distributed training procedure described above, except that within the four blocks stimuli were delivered at a 10 -sec ISI rather than a 60 -sec ISI. There were no significant differences between trained and untrained worms on test day when a 10-sec ISI was employed with the group-training protocol with either massed $(t(39)=1.03, P>0.10)$ or distributed training $(t(41)=0.53, P>0.10$; Fig. $2 \mathrm{~A})$ when each was compared with its tap control group. Therefore, this experiment replicated Beck and Rankin (1997) by showing that no long-term memory for habituation was produced with the group-training protocol when the stimuli were delivered at a 10-sec ISI.

Beck and Rankin (1995) reported that long-term memory for distributed habituation training with the single worm protocol could be blocked if heat shock was administered in the 1-h interval between blocks of training. They hypothesized that heat shock stopped ongoing protein synthesis and blocked new protein synthesis from occurring for a critical period of time after each training block. In this experiment, the effects of heat shock on the new group-training protocol were tested. There were four groups in this experiment; the first received

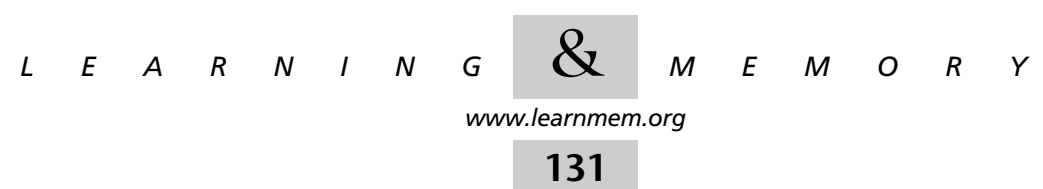




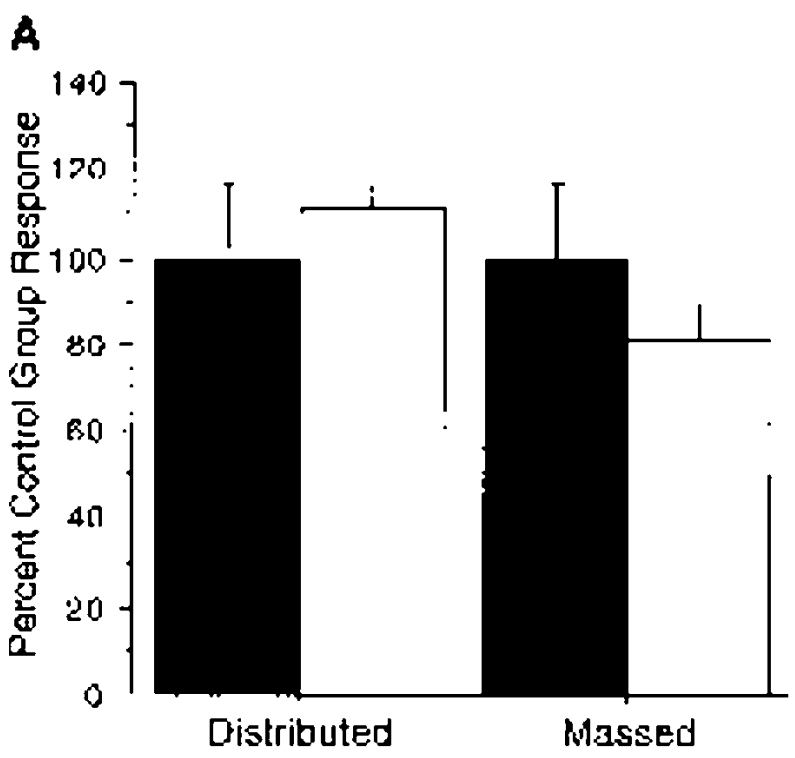

B

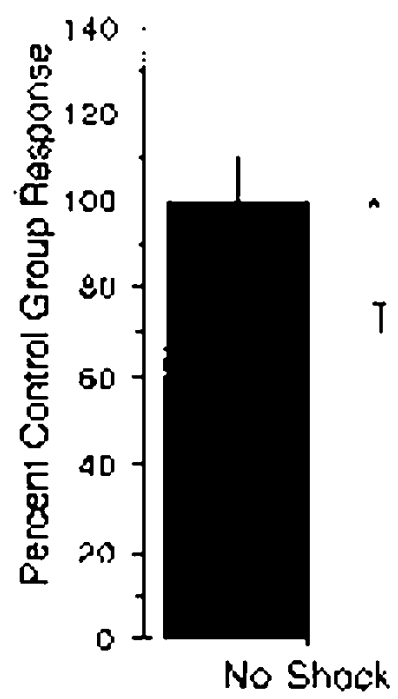

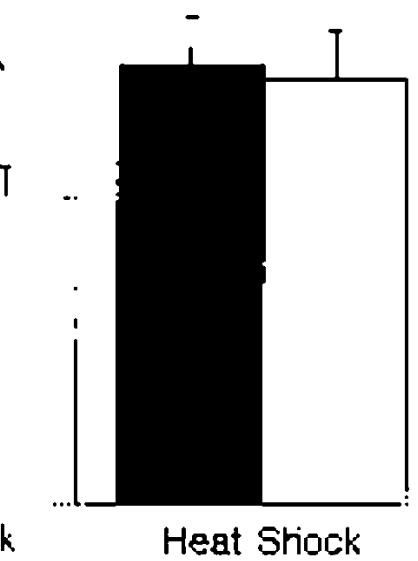

Figure 2 The new group-training protocol replicates previous findings that distributed training at a short ISI does not produce long-term memory for habituation training $(A)$ and that heat shock given during the rest periods that separate the distributed training blocks eliminates long-term memory $(B)$. (A) The mean response magnitude for trained groups (open bars) that received habituation training at a 10-sec ISI using either a massed or distributed training protocol compared with their respective single-tap matched control group (solid bars). Mean response magnitudes are expressed as percent control group response. There was no difference in average response magnitude between either pair of trained and control groups. (B) Mean response magnitudes for groups that received distributed training with heat shock given in the first $40 \mathrm{~min}$ of each 1-h rest period (open bar) do not differ from mean response magnitudes of the matched control group that also received heat shock (solid bar). A control condition where trained worms received distributed training and then underwent $21^{\circ} \mathrm{C}$ (room temperature)-shock during the rest periods between training blocks (open bar) show significantly decreased mean response magnitudes compared with its matched control group (solid bar). Mean response magnitudes are expressed as percent control group response. $\left(^{*}\right) P<0.05$. the normal distributed training protocol with no heat shock treatment (no-shock group), the second was its tap control group. The third group received the distributed training protocol, and was given heat shock treatment for $40 \mathrm{~min}$ following each of the four training blocks (heat-shock group). The fourth group was tap control for the third group, and received the same exposure to heat shock as the trained group, but only a single tap on day 1 . The results showed normal long-term memory in the no-shock-treatment training group $(t(45)=2.52, P<0.05)$ and, in the heatshock group, heat shock blocked the long-term memory for habituation normally produced by the group-training proto$\operatorname{col}(t(34)=0.18, P>0.10$; Fig. 2B).

Taken together, these experiments replicate the principle findings of Beck and Rankin (1995, 1997) concerning long-term memory for habituation training in C. elegans. We can now use this new, more rapid protocol to further explore the characteristics and mechanisms of long-term memory in C. elegans.

\section{Shortening the Inter-Block Interval Decreased Long-Term Memory}

Beck and Rankin (1995) showed that heat shock effectively blocks long-term memory formation only when it is administered during the first $30 \mathrm{~min}$ of the $1-\mathrm{h}$ rest periods. With this in mind, we tested whether we could further shorten the training procedure with the new group-training protocol by running a distributed training group with only 40-min rest intervals between blocks of training. When the interblock interval was only $40 \mathrm{~min}$, there was no significant long-term memory for distributed training as the average response magnitude for the trained group was $86 \%$ of the average control response (mean for 18 trained worms was $168.82 \pm 19.45$, for 21 tap control worms was $196.08 \pm 16.53$; $t(37)=1.08, P=0.29 \mathrm{NS})$. Therefore, in all future experiments using this procedure, the inter-block interval must be at least $60 \mathrm{~min}$.

\section{The New Group Protocol Shows an Accumulation of Memory With Increased Training}

Using nonsense word lists with human subjects, Ebbinghaus (1885) demonstrated that information is remembered longer if it is studied in blocks across time rather than all at once. The degree to which information was retained corresponded with the number of learning trials; greater retention occurred as the number of learning trials increased. As we have shown, in C. elegans, only distributed training results in long-term memory $24 \mathrm{~h}$ after training. If the principles outlined by Ebbinghaus apply to long-term memory for habituation in the worm, then as more training blocks are included in the training procedure a greater degree of long-term retention should be produced. To test this, four groups of worms received either one $(n=20)$, two $(n=22)$,

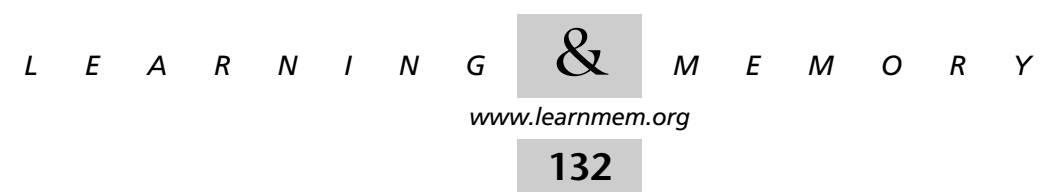


three $(n=20)$ or four blocks $(n=23)$ of 20 single taps at a 60 -sec ISI all separated with 1 -h rest periods. Each experimental training group was coupled to a single-tap matched control group ( $n=21,22,17$, and 23 , respectively).

Figure 3 shows the standardized overall mean reversal response magnitudes for the 10 test single-taps for experimental and control groups and illustrates the graded increase in retention that was observed as the number of training blocks increased. Using $t$-tests with the $\alpha$ level corrected for multiples tests, it was found that neither one, two, nor three blocks of habituation training produced significant long-term memory $(t(39)=0.84, P>0.10, t(42)=1.68$, $P>0.10$, and $t(35)=1.87, P>0.10$, respectively). Four blocks of habituation training, however, produced the greatest difference between the trained group and the matched singletap control $(t(44)=2.67, P<0.05)$. These results demonstrate that memory accumulates as the number of training blocks increases. As well, this experiment supports the conclusion that the four blocks used in the new group-training protocol is an optimal balance between the time taken in training and the amount of long-term memory produced to make the new protocol an effective tool in exploring cellular mechanisms of long-term memory.

\section{Presynaptic Glutamate Release Is Required to Produce Long-Term Memory}

Wicks and Rankin (1995) mapped the neural circuit responsible for the reversal response a worm exhibits following a tap and have identified the synapse between the sensory neurons and the inter-neurons as the likely locus of plasticity (Wicks and Rankin 1997). There is evidence from studies of identified mutations of genes expressed in pre- and post-

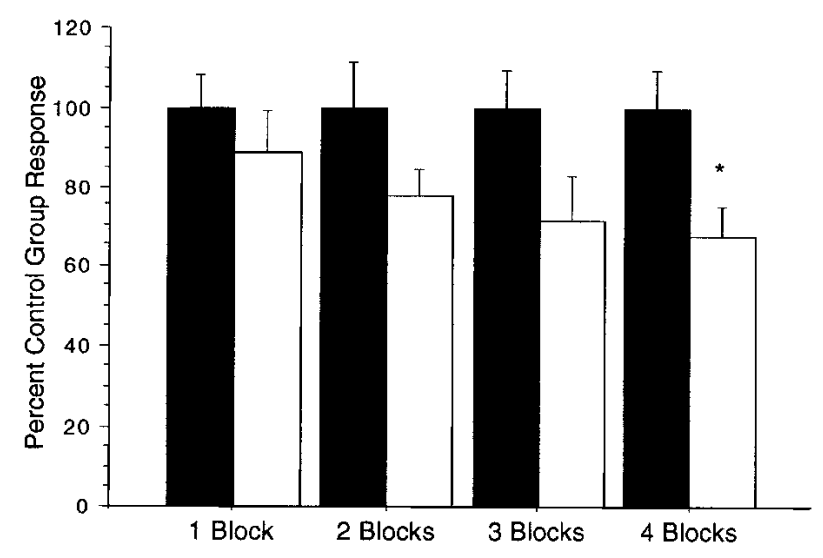

Figure 3 Memory appears to accumulate as an increased number of training blocks are added. Mean response magnitudes of test phase for trained groups that received one, two, three, or four blocks of training (open bars) compared with their respective single-tap matched control groups (solid bars) shows that as amount of training increases, the mean response magnitude decreases for the trained group, therefore indicating greater long-term retention. Mean response magnitudes are expressed as percent control group response. $\left({ }^{*}\right) P<0.05$. synaptic neurons in the tap-withdrawal circuit that these sensory to inter-neuron synapses are glutamatergic. For example, the mammalian homolog of EAT- 4 is a vesicular glutamate transporter and in C. elegans EAT- 4 is expressed in the sensory neurons of the tap withdrawal circuit (Lee et al. 1999). Lee et al. (1999) hypothesized that in C. elegans, EAT-4 has a major role in regulating the synthesis of the neurotransmitter glutamate. The eat- 4 mutation does not disrupt the worm's ability to reverse spontaneously or in response to a single tap. In a study of short-term habituation with both short and long ISIs, however, worms with a lossof-function, putative null mutation in eat- 4 showed more rapid habituation to tap and slower recovery from habituation than did wild-type worms (Rankin and Wicks 2000).

In addition, genes have been isolated that are expressed on the inter-neurons of the tap withdrawal circuit that code for three classes of glutamate receptors: $g l r-1$ (homologous to AMPA type; Hart et al. 1995; Maricq et al. 1995), avr-15 and avr-14 (glutamate-gated Cl-channels; Dent et al. 1997, 2000), and $n m r-1$ (homologous to NMDAtype channels; Brockie et al. 2001).

We examined the role of presynaptic glutamate release in the formation of long-term memory for habituation training by studying eat- 4 worms. If the deficit in sustained glutamate activity that was seen in short-term habituation negatively affects memory formation, it would strongly indicate that glutamate transmission is necessary for long-term memory for habituation training in the tap-withdrawal circuit. In the first experiment, eat-4(ky5) worms were tested using the original single-worm protocol with three blocks of trains of taps at a 60-sec ISI. In this protocol, it is possible to examine the acquisition of memory by looking at performance during training. Figure $4 \mathrm{~A}$ shows the average response magnitudes for reversals in the three blocks of training on day 1 and the test block on day 2. When stimulated with trains of taps, the eat- 4 worms showed normal shortterm habituation over each block of stimuli. With trains of taps, eat-4 worms do not show the rapid and complete habituation reported by Rankin and Wicks (2000) for single taps. In addition, as can be seen in Figure $4 \mathrm{~B}$, there was a steady accumulation of short-term habituation over the course of the three training blocks on day 1 (similar to that reported in wild-type worms by Beck and Rankin 1997). When the mean response magnitude for the first block of training was compared with the mean of the test block on day 2, there was no statistically significant long-term memory for habituation, however, there was a trend for smaller responses on the test day $(t(18)=1.64, P=0.12)$. Also, day 2 habituation appears to be more rapid compared with the habituation rates on day 1 with the largest drop in reversal magnitude occurring between stimuli 1 and 2; this more rapid rehabituation may reflect memory for the earlier training (Fig. 4A).

In a second experiment, eat- 4 worms were tested us-

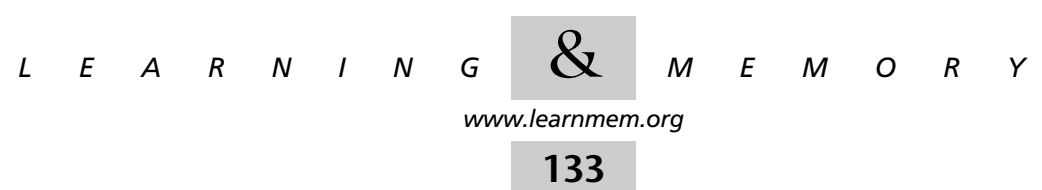




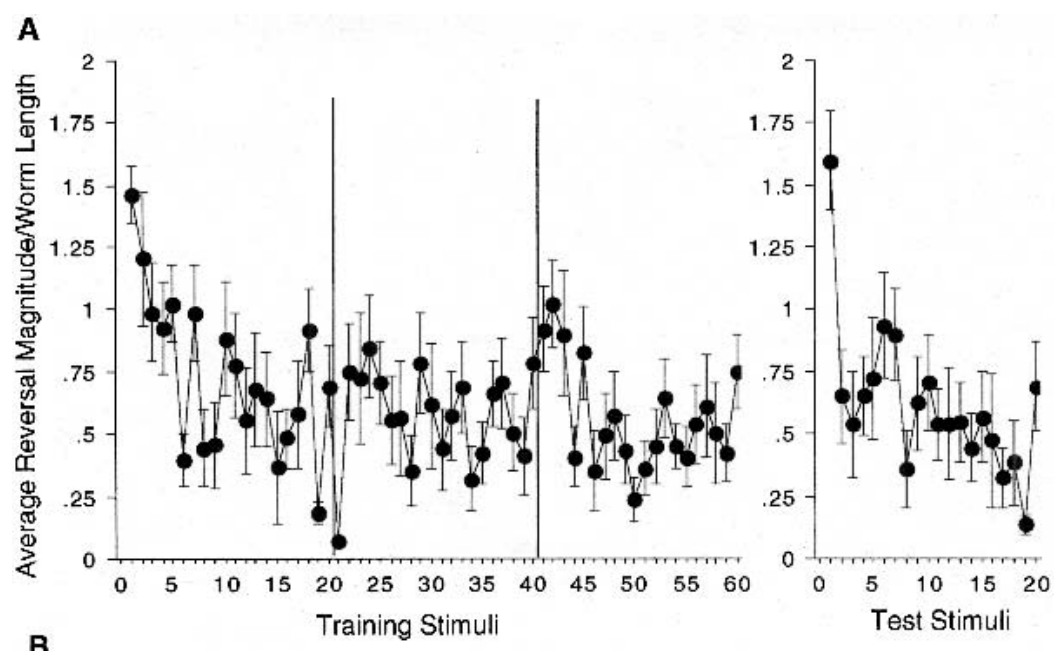

B

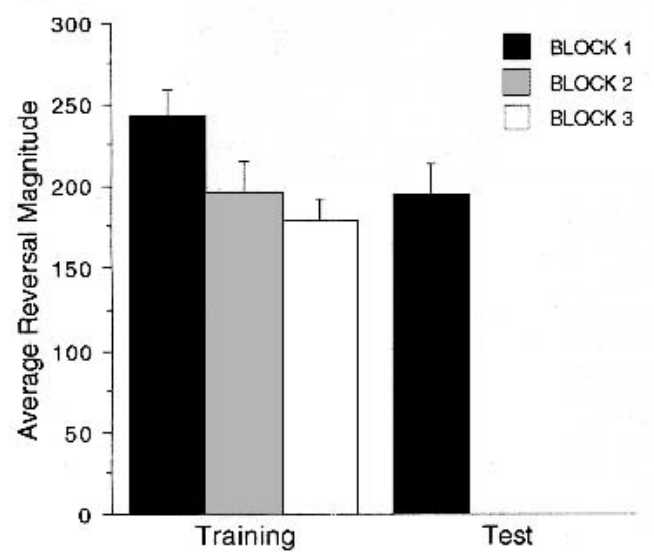

Figure 4 With trains of taps, eat-4 mutant worms show significant short-term accumulation of habituation across blocks of training and a trend toward 24-h retention. ( $A$ ) The mean response magnitude, standardized by worm length, for each train stimulus is graphed across all 20 stimuli in each of the three training blocks and across the 20 stimuli of the test block that occurred $24 \mathrm{~h}$ later. (B) Average reversal magnitude for each training block on day 1 and the test phase on day 2 .

ing the new group-training method where the distributed group received four blocks of single taps. When the mean reversal magnitudes to the 10 test single-taps were compared between the trained group and their single-tap controls $(t(42)=0.38, P=0.71$; Fig. 5$)$, the eat -4 worms showed no evidence of long-term memory for the habituation to tap training.

To verify that it was the eat- 4 mutation that produced the deficit in long-term memory following training with single taps and not some secondary mutation associated with the eat- 4 mutant strain, a transgenic eat- 4 rescue strain (DA1242 in which a sequence including the eat-4 gene has been added back into eat- 4 worms) was given distributed training using the new group-training protocol with single taps. The DA1242 strain was originally described by Lee et al. (1999) and, in behavioral assays, was found to restore not only the feeding deficits originally reported for eat-4 worms, but also the change in short-term habituation reported by Rankin and Wicks (2000). When DA1242 was tested for longterm memory for habituation, the trained group showed significantly smaller responses than the did the single-tap controls $(t(47)=2.70, P<0.05$; Fig. 5$)$. This indicated that the eat- 4 transgene successfully rescued the long-term memory for habituation training with single taps and that the deficit seen in eat- 4 worms was the result of a mutation in eat-4 and not in some other unknown gene.

One major difference between the original single-worm protocol and the group-training protocol is that the original protocol used trains of taps as the stimulus, whereas the new protocol used a single tap as the stimulus. Rankin and Wicks (2000) reported very rapid habituation in eat-4 worms with both short and long ISIs using the single-tap stimulus. In contrast, in the single-worm protocol when trains of taps were delivered at a 60 -sec ISI, the rate of habituation over 20 stimuli for eat-4 worms was only marginally faster than what we see in wild-type worms (i.e., Beck and Rankin 1997). Therefore, it appeared that eat-4 worms showed less of an alteration in shortterm habituation to trains of taps than to single-tap stimuli. When the results from the original protocol with three blocks of 20 trains of taps $(t(18)=1.64, P=0.12)$ are compared with the results of the new protocol with four blocks of 20 single taps $(t(42)=0.38, P=0.71)$ it appears as though the trains of taps might be producing some memory, whereas it is clear that the single taps are not. To test whether trains of taps could produce long-term memory for habituation, the new group-training protocol was given using four trains of taps rather than single taps. Using the new group-training procedure with the stronger train of taps resulted in significant long-term memory for habituation training in the group of eat-4 mutants that received distributed training $(t(34)=2.54, P<0.05)$ when compared with the single train control group (Fig. 5). Therefore, if sufficient stimulation is given, the eat- 4 worms will show long-term memory for habituation training.

\section{DISCUSSION}

From these experiments it can be seen that the new grouptraining protocol for studying long-term memory for habituation in C. elegans is as effective as the original protocol reported in Beck and Rankin (1997). Both paradigms pro-

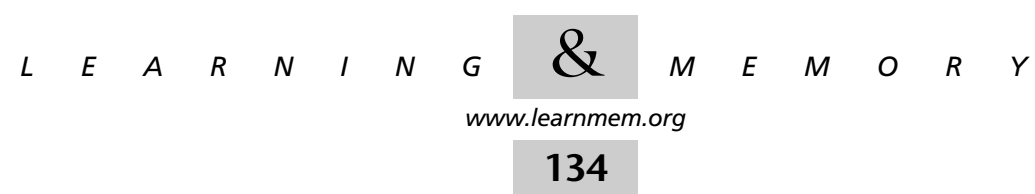




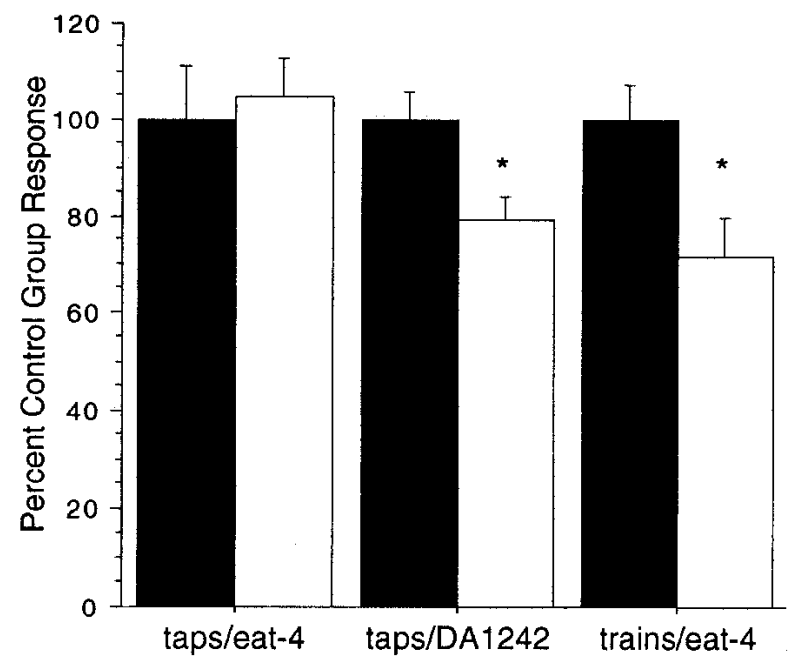

Figure 5 With the new group-training protocol 24-h retention occurs with eat- 4 worms only if a strong stimulus (trains) is applied. The mean response magnitudes for each trained group (open bars) are expressed as percent control response (solid bars). When the weaker tap stimulus is used for training, no 24-h retention results when compared with the matched control group. The eat- 4 rescue strain (DA1242), where the eat-4 mutant is rescued by a wild-type transgene, shows long-term memory for habituation with single taps rescuing the deficit. If a stronger stimulus (trains) is used with the new group-training protocol, long-term memory for habituation is also produced. $\left(^{*}\right) P<0.05$.

duced results that showed that distributed training resulted in long-term memory, whereas massed training did not; that long ISIs produce long-term memory, whereas short ISIs did not; and that heat shock during training blocked long-term memory for habituation. Because groups of worms can be tested within days (as opposed to weeks and months with the original single-worm protocol), the new group-training protocol provides a faster, alternative method with which to examine long-term memory effectively.

Many researchers using other organisms have reported that learning is retained for longer periods of time as subjects are exposed to more and more blocks of training during the learning phase (i.e., Tully and Quinn 1985). When the degree of long-term memory was examined across groups that received one, two, three or four blocks of training, it is evident that the amount of memory gradually increased as another block of training was added. Because massed training did not produce long-term memory, it appears that the rest periods between training blocks are of importance for memory formation. Like Beck and Rankin (1995), we report that heat shock administered during the rest periods between training blocks disrupts long-term memory formation. Heat shock produces a sudden yet sustained state of stress in an organism that causes all nonessential protein synthesis to cease, including de novo protein synthesis thought to underlie long-term memory (Schlesinger et al. 1982; Lindquist 1986). The disruption of long- term memory formation by heat shock indicates that some sort of consolidation of memory occurs during the $1 \mathrm{~h}$ between training blocks and that this consolidation process is dependent on protein synthesis. The results from the accumulation experiment indicate that at least three blocks and/ or two rest periods are necessary for this protein synthesis to occur. The length of the rest periods is also important; when the rest period was shortened to $40 \mathrm{~min}$, long-term memory for habituation was not observed. Although Beck and Rankin (1995) suggested that the first 30 min posttraining was critical for protein synthesis, the results from this experiment indicate some additional function for the 60-min rest period. It would also be interesting to investigate the maximum duration of the rest period that still supports long-term memory for habituation to develop.

Using a simple system like $C$. elegans, where the neurons and synapses of a particular learned behavior have been mapped, allows us the opportunity to examine the neurotransmitters and receptors that are necessary for longterm memory formation. Many mutant strains are available in $C$. elegans for behavioral analysis. The synapse between the tap sensory neuron and the inter-neurons is hypothesized to be glutamatergic (Rankin and Wicks 2000). When a mutation in eat-4, a vesicular glutamate transporter, was present, the amount of glutamate available at the sensory neuron terminal was apparently decreased and short-term habituation was very rapid. In eat-4 worms, distributed training with a tap stimulus did not produce long-term memory. This indicated that sustained glutamatergic activity is necessary for long-term memory formation.

The observation that distributed training with stronger stimulation produced long-term memory progressively in eat-4 worms indicates that if enough strong stimuli are used, sufficient glutamate is released from the sensory neuron to produce long-term memory for habituation training: four blocks of single taps produced no long-term memory, whereas three blocks of trains of taps resulted in a trend toward long-term memory but it took four blocks of trains of taps for eat-4 to show long-term memory. One possibility is that the high frequency train of taps might stimulate the sensory neurons to release a second neurotransmitter, however, this hypothesis is not supported by other experiments that indicate that activation of a C. elegans homolog of the AMPA-type glutamate receptor ( $g l r-1)$ is necessary for longterm memory for habituation (J. Rose, S. Chen, K. Kaun, and C. Rankin, unpubl.).

This study has examined long-term memory for habituation of wild-type worms and mutant eat-4 worms using either the original single-worm training procedure or the new group-training procedure. Both protocols produced comparable results, thereby supporting a more efficient way of examining the characteristics of long-term memory in C. elegans. The effect of increased stimulation leading to increased long-term memory for habituation training in

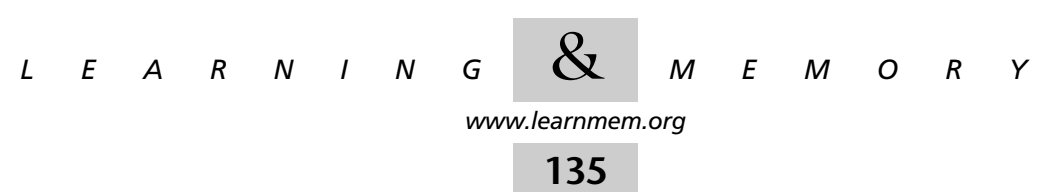


eat -4 worms indicates that glutamate does participate in the formation of long-term memory in the worm. This finding sets the stage for studies of the role of mutations in a variety of genes affecting glutamate transmission on learning and memory.

\section{MATERIALS AND METHODS}

\section{Subjects}

A total of 469 hermaphroditic C. elegans Bristol (N2), 99 eat-4 (ky5), and 50 DA1242 were used. N2 worms were originally obtained from the Caenorhabditis Genetics Center. eat- 4 and the eat- 4 transgenic rescue strain DA1242 were originally obtained from the laboratory of Dr. Leon Avery and the University of Texas Medical School. eat-4(ky5) is a loss-of-function, putative null allele of a glutamate vesicular transporter (Avery 1993). Experiments using the wild-type strain and eat-4 had between 18-27 worms per group. Lee et al. (1999) showed that the eat- 4 gene rescuing activity was localized to a $6.9-\mathrm{kb}$ region of cosmid ZK512. The strain (DA1242) was made using cosmid rescue with cosmid ZK512 that restored the wild-type feeding phenotype. Rankin and Wicks (2000) showed that DA1242 also rescued the short-term habituation deficits seen in eat-4 worms. All worms were maintained on Nematode Growth Medium (NGM) agar seeded with Escherichia coli (OP50) as described in Brenner (1974).

\section{Behavioral Testing}

Habituation training and testing took place under a stereomicroscope (Wild M3Z, Wild Leitz Canada) connected to a digital camera (Panasonic Digital 5100), VCR (Panasonic AG1960), and monitor (NEC). In all recorded conditions, a time-date generator (Panasonic WJ-810) superimposed the time-date on the video record in addition to a digital clock that counted to the hundredths of a second. If the original training protocol was employed, then the training phase occurred with the microscope stage light on to allow for video-recording of responses. In the new protocol, the training was not recorded, therefore the training phase occurred without the microscope stage illuminated. Testing for both protocols occurred with the stage light on and was video-recorded. Habituation stimuli for both protocols were administered via electrical stimulation from a Grass $\$ 88$ stimulator resulting in a mechanical tapper exerting 1-2 $\mathrm{N}$ of force to the side of the petri plate on which worms were kept producing a single tap stimulus. This set-up could also be used to administer the stronger "train of taps" stimulus that consists of a $10-\mathrm{Hz}$ train of six taps.

The original distributed training protocol to produce longterm memory for habituation training (described in detail in Beck and Rankin 1997) consisted of an experimental group in which individual 4-d-old worms received three blocks of training. Each block consisted of 20 trains of taps given at a 60-sec ISI. Training blocks were separated by 1 -h rest periods on day 1 , and a single block of 20 trains of taps was given $24 \mathrm{~h}$ later on day 2 . To test for long-term memory for habituation, the mean reversal magnitude to the first block of trains of taps on day 1 was compared with the mean reversal magnitude for the block of trains of taps on day 2. A significantly smaller mean response on day 2 was considered to reflect long-term memory for habituation training. With this procedure, training and testing of 20 worms took $\sim 67 \mathrm{~h}$.

In comparison, the group-training distributed protocol consisted of an experimental group of 15-20 4-d-old worms trained simultaneously on a single plate with four blocks of training. Each block consisted of 20 single-tap stimuli given at a 60-sec ISI with 1-h rest periods between blocks. Single taps were used in this protocol to shorten the time required to score the video-recorded data. A control group that included another 15-20 worms received only a single tap on training day. At least $1 \mathrm{~h}$ after the last tap of training, worms from both the trained and single-tap control were transferred onto labeled, individual E. coli-streaked plates. Worms from both groups were tested one at a time with 10 single taps given at a 60-sec ISI: testing occurred $22-28 \mathrm{~h}$ following training (median $>24 \mathrm{~h}$ ).

In the original procedure, memory was measured by comparing the 20 responses of the test day with the first 20 responses on the training day. The new group-training protocol does not allow for gathering of data during the training phase. Therefore, in this protocol, memory is measured as a comparison of average response magnitude to the 10 test single taps between the trained group and an untrained matched control group. Long-term memory for habituation training was measured as a significant difference using a between-groups comparison for the test responses for the experimental and control groups. With this protocol it took $\sim 14 \mathrm{~h}$ to train and test 20 experimental and 20 control worms.

For massed training in the new protocol, an experimental group of 15-20 worms received one block of 80 stimuli at a 60 -sec ISI on training day and a control group of 15-20 worms received only a single-tap stimulus. At least $1 \mathrm{~h}$ after the last tap of training, worms from both the trained and single-tap control were transferred onto labeled, individual plates. Twenty-four hours later, both groups were tested one at a time with 10 single taps given at a 60-sec ISI. The mean reversal magnitude across the 10 test single taps was compared between the two groups. Long-term memory for habituation training was measured as a significant difference between the trained and control groups on test day.

\section{Scoring}

Video records of the training and testing phases (original protocol) or of the test phases of the trained and single-tap control group (new protocol) were scored using stop-frame video analysis onto acetate sheets. These tracings of reversal magnitude were then scanned into a computer and measured using NIH image software. Previous research has found that $90 \%$ of the time, adult worms respond to a single tap or train of taps with a reversal (Chiba and Rankin 1990), therefore, it was expected that worms would respond a majority of the time to a single tap or train of taps with a reversal. Over the course of an experiment, however, a worm occasionally accelerated forward to tap. The neural circuit for accelerations forward is different from the neural circuit for reversals to tap, and habituates with different kinetics (Wicks and Rankin 1995, 1996). Therefore, accelerations were considered qualitatively different from reversals and were scored as missing data points. Fewer than $10 \%$ of the data points fell into this category. If the worm paused in response to the tap/train or did not respond to the stimulus, then this was scored as zero.

\section{Data Analysis}

All data analyses were conducted using Statview 4.5. All reversal magnitude values were averaged across the test phase. When responses to individual stimuli across the test phase are shown, reversal magnitudes were standardized by dividing them by the worm length of the respective worm. In the single-worm protocol, two-tailed paired $t$-tests were used to compare the first block of training with the test block on day 2 . In the group-protocol, the

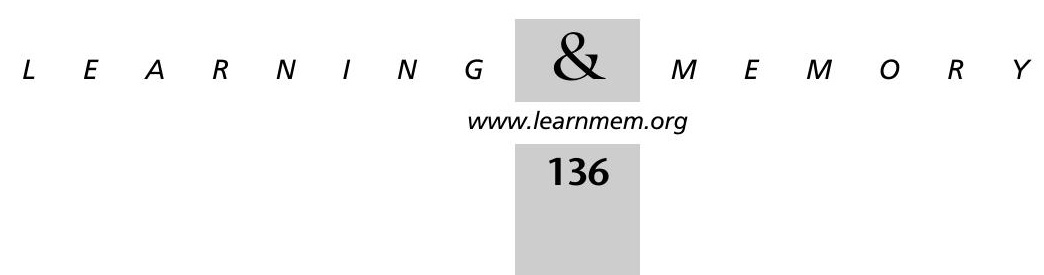


responses of experimental and control groups were compared using a two-tailed unpaired $t$-test. Where more than two groups were compared, $t$-tests were used with a Bonferonni adjustment of the $\alpha$ level for multiple $t$-tests.

\section{Heat Shock Treatment}

In an experiment in which worms received heat shock treatment, both the trained group and the matched tap control group underwent heat shock. Heat shock was given by double-wrapping petri plates with parafilm on the training day and submerging them in a $32^{\circ} \mathrm{C}$ bath for the first $40 \mathrm{~min}$ of each 1-h rest period. During the last $20 \mathrm{~min}$ of the 1-h rest periods, training plates were returned to room temperature. For comparison, the no-shock control group underwent the same procedure except that plates were submerged in a room temperature $\left(21^{\circ} \mathrm{C}\right)$ bath.

\section{ACKNOWLEDGMENTS}

We thank Amelia Wan for scoring some of the experiments reported here. This work was funded by an Natural Sciences and Engineering Research Council of Canada (NSERC) operating grant to C.H.R.; an NSERC PGSB to J.K.R.; and an NSERC summer fellowship to K.R.K.

The publication costs of this article were defrayed in part by payment of page charges. This article must therefore be hereby marked "advertisement" in accordance with 18 USC section 1734 solely to indicate this fact.

\section{REFERENCES}

Avery, L.1993. The genetics of feeding in Caenorbabditis elegans Genetics 133: 897-917.

Beck, C.D.O. and Rankin, C.H. 1995. Heat-shock disrupts long-term memory consolidation in Caenorhabditis elegans. Learn. Mem. 2: $161-177$.

. 1997. Long-term habituation is produced by distributed training at long ISIs and not by massed training or short ISIs in Caenorbabditis elegans. Anim. Learn. Behav. 25: 446-457.

Brenner, S. 1974. The genetics of Caenorhabditis elegans. Genetics 77: 71-94.

Brockie, P.J., Mellem, J.E., Hills, T., Madsen, D.M., and Maricq, A.V. 2001. The C. elegans glutamate receptor subunit NMR-1 is required for slow NMDA-activated currents that regulate reversal frequency during locomotion. Neuron 31: 617-630.

Carew, T.J., Pinsker, H.M., and Kandel, E.R. 1972. Long-term habituation of a defensive withdrawal reflex in Aplysia. Science 175: 451-454.

Chiba, C.M. and Rankin, C.H. 1990. A developmental analysis of spontaneous and reflexive reversals in the nematode Caenorhabditis elegans. J. Neurobiol. 21: 543-554.
Dent, J.A., Davis, M.W., and Avery, L. 1997. avr-15 encodes a chloride channel subunit that mediates inhibitory glutamatergic neurotransmission and ivermectin sensitivity in Caenorhabditis elegans. EMBO J. 16: 5867-5879.

Dent, J.A., Smith, M.M., Vassilatis, D.K., and Avery, L. 2000. The genetics of ivermectin resistance in Caenorhabditis elegans. Proc. Natl. Acad. Sci. 97: 2674-2679.

Ebbinghaus, H. 1885. Retention as a function of repeated learning. In Memory. Reprint ed., 1964. pp. 81-89. Dover, New York.

Groves, P.M. and Thompson, R.F. 1970. Habituation: A dual-process theory. Psychol. Rev. 77: 419-450.

Hart, A.C., Sims, S. and Kaplan, J.M. 1995. Synaptic code for sensory modalities revealed by $C$. elegans GLR-1 glutamate receptor. Nature 378: 82-85.

Lee, R.Y., Sawin, E.R., Chalfie, M., Horvitz, H.R., and Avery, L. 1999 EAT-4, a homolog of a mammalian sodium-dependent inorganic phosphate co-transporter, is necessary for glutamatergic neurotransmission in Caenorhabditis elegans. J. Neurosci. 19: 159-167.

Lindquist, S. 1986. The heat-shock response. Annu. Rec. Biochem. 55: 1151-1191.

Maricq, A.V., Peckol, E., Driscoll, M., and Bargmann, C.I. 1995. Mechanosensory signaling in C. elegans mediated by the GLR-1 glutamate receptor. Nature 378: 78-81.

Rankin, C.H. and Wicks, S.R. 2000. Mutations of the Caenorbabditis elegans brain-specific inorganic phosphate transporter eat-4 affect habituation of the tap-withdrawal response without affecting the response itself. J. Neurosci. 20: 4337-4344.

Schlesinger, M., Tissieres, A., and Ashburner, M. 1982. The effects of heat shock and other stress on gene activity: An introduction. In Heat shock proteins: From bacteria to man. (eds. M. Schlesinger, A. Tissieros, and M. Ashburner) pp. 1-9. Cold Spring Harbor Laboratory, Cold Spring Harbor, NY.

Tully, T. and Quinn, W.G. 1985. Classical conditioning and retention in normal and mutant Drosophila melanogaster. J. Comp. Physiol. [A] Sensory Neural Behav. Physiol. 157: 263-277.

Tully, T., Preat, T., Boynton, S.C., and Del Vecchio, M. 1994. Genetic dissection of consolidated memory in Drosophila. Cell 79: 35-47.

Wicks, S.R. and Rankin, C.H. 1995. Integration of mechanosensory stimuli in Caenorhabditis elegans. J. Neurosci. 15: 2434-2444.

- 1996. Recovery from habituation in Caenorhabditis elegans is dependent on interstimulus interval and not habituation kinetics. Behav. Neurosci. 110: 840-844.

. 1997. Effects of tap withdrawal response habituation on other withdrawal behaviors: The localization of habituation in the nematode Caenorhabditis elegans. Behav. Neurosci. 111: 342-353.

Received January 22, 2002 ; accepted in revised form March 28, 2002. 


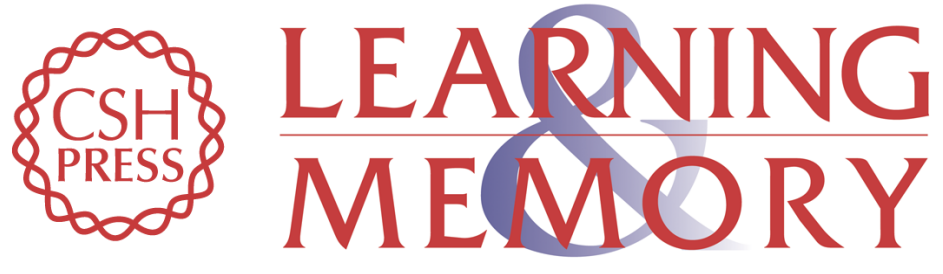

\section{A New Group-Training Procedure for Habituation Demonstrates That Presynaptic Glutamate Release Contributes to Long-Term Memory in Caenorhabditis elegans}

Jacqueline K. Rose, Karla R. Kaun and Catharine H. Rankin

Learn. Mem. 2002, 9:

Access the most recent version at doi:10.1101//m.46802

References This article cites 19 articles, 9 of which can be accessed free at: http://learnmem.cshlp.org/content/9/3/130.full.html\#ref-list-1

License

Email Alerting Receive free email alerts when new articles cite this article - sign up in the box at the Service top right corner of the article or click here. 